\title{
Analysis on the Application Effect of Psychological Counseling for COVID-19 Patients
}

\author{
Lincy Issac ${ }^{1}$, Deepak K Nair ${ }^{2}$ \\ ${ }^{1}$ Assistant Professor, MOSC College of Nursing \\ ${ }^{2}$ Assistant Professor, MOSC College of Nursing
}

\begin{abstract}
The COVID-19 outbreak is a global emergency. The consequences of the pandemic were numerous. Mental health and psychosocial support services have been severely impacted by the COVID-19 pandemic. Innovative solutions such as tele-consultations, digital self - help platforms, novel approaches to ensure supply of psychotropic medicines, and task sharing/shifting for basic psychosocial support, are being used to defeat service disruptions and maintain care for those with psychological conditions.
\end{abstract}

Keywords: Psychosocial counseling, Skills, COVID - 19

\section{Introduction}

COVID-19, a contagious disease, has stoked fears among people across nations due to its severe morbidity, mortality and potency of high transmission. This has caused severe anxiety among people who fear the possibility of getting them infected as well as passing on their infection to friends, families and co - workers. Persons with pre - existing mental health discomfort may feel more distressed and that can worsen their health. Psychosocial counseling is the first line care provided to persons in the form of emotional support in a culturally suitable way.

Who can provide support in counseling?

- Professional Counselors

- Post Graduate in Psychology or Social Work having adequate counseling experience

- Health care workers trained in counseling

- Trained volunteers who render services to support the COVID-19 patients

Rules to be followed for Psychosocial Counseling:

- Maintain a good relationship with affected persons

- Gain confidence of the needy persons.

- Ensure confidentiality of the interactions.

- Counselor should show empathy.

- Give importance to client's opinion and viewpoints.

\section{Goals of Counseling}

Effective counseling helps clients to enhance skills needed to cope up with crisis such as COVID-19 by way of improved decision making and behaviour changes.

1) Create awareness about safe practices on health and hygiene.

2) Alleviate distress.

3) Advise clients to take on the worries in one's stride by developing healthy ways of addressing worry.

4) Suggest practical steps towards solving life problems.

5) Encourage open and positive communication in relationships.

6) Help clients to cope better with life challenges.

7) Create a feeling of realistic hope.

\section{Counseling Skills}

Skills that a counselor needs to possess are the following:

- Personally attending: A family will immensely benefit if their concerns are attended to with utmost patience and respect. Statements like "I am here to listen to you", "I would like to know more about what your concerns are" would certainly comfort the client and family.

- Active and focused listening

- Probing using open - ended questions

- Exuding compassion

- Assuring confidentiality

- Using simple language

- Psycho - educating: This involves providing basic facts, information and reliable resources to the clients regarding their concerns.

- Assessing risks like suicidal tendencies, violence or abuse and adopt appropriate safety and crisis management

- Dealing with issues of stigma and discrimination, if any

- Maintain contacts of referrals like Police, Medical, De addiction support etc and provide to client and/or family on a need basis and arrange to connect with other local support services, if necessary

- Problem - solving: Counselors must set out a clear process for solving or alleviating the concerns of the client

- Create realistic hope and strengthen resilience

What Support Will Be Provided

- Confidentiality and privacy for interactions

- Emotional support

- Access to reliable and helpful information based on the knowledge level

- Referral to medical and mental health

- Referral to other support services

\section{Qualities of an Effective Counselor}

The qualities of a good Counselor go hand in hand with good counseling skills.

- Warmth: Facilitate a conducive environment for clients to share their deepest fears and anxieties 
- Acceptance: Promise of accepting Client's story with unconditional positivity

- Flexibility: Adapt to client's needs with an open perspective

- Stay updated: Awareness of latest happenings, medical research

- Contextual understanding: To customise the counseling needs based on the socioeconomic, cultural and background of client.

\section{Algorithm for Counselors}

Pre Counseling - Understand the general principles of counseling, develop good communication skills, cultivate active listening skills, respect for privacy and confidentiality Step 1: Establish Rapport

Step 2: Provide information about the illness and testing process

Step 3: Listen to the worries with utmost attention

Step 4: Address the mental health concerns

Step 5: Clarify the myths about COVID-19

Step 6\& 7: Enhance self - esteem and reduce stigma

Step 8: End the session with reassurance

Specific Principles To Provide Psychosocial Counseling for Covid Positive Patients

\section{Establish a Therapeutic Relation:}

A strong therapeutic relationship is recommended to overcome the varied mental conditions (apprehensions, anxieties, misconceptions, fears etc) that the patients undergo. The most recommended way for developing good relationship with the patients is though a proper self introduction of the Counselor and then giving clarity on the reason behind the visit as well as goal of the counseling, followed by initiating an open communication with the patients about their life in a manner they want to describe.

Quick Assessment: Pre - Requisite to Intervention

a) Assessment of mental health necessitates a close observation of the predominant mood state of the patient (sadness, fear, anxious, anger etc), assessment of the severity of the mood state (mild, moderate or severe), estimation of individual's ability to deal with it (high or low self - efficacy) and perception of psychological support in life (present or absent).

b) The stages of the disease and its recovery can be classified into three broad phases as mentioned in the below figure.

Through the active intervention of the Counselor, specific needs of each phase may be incorporated sensitively. The assessment helps to arrive at the extent and nature of psychosocial intervention that varies across the three phases, with each having its own goal and techniques.

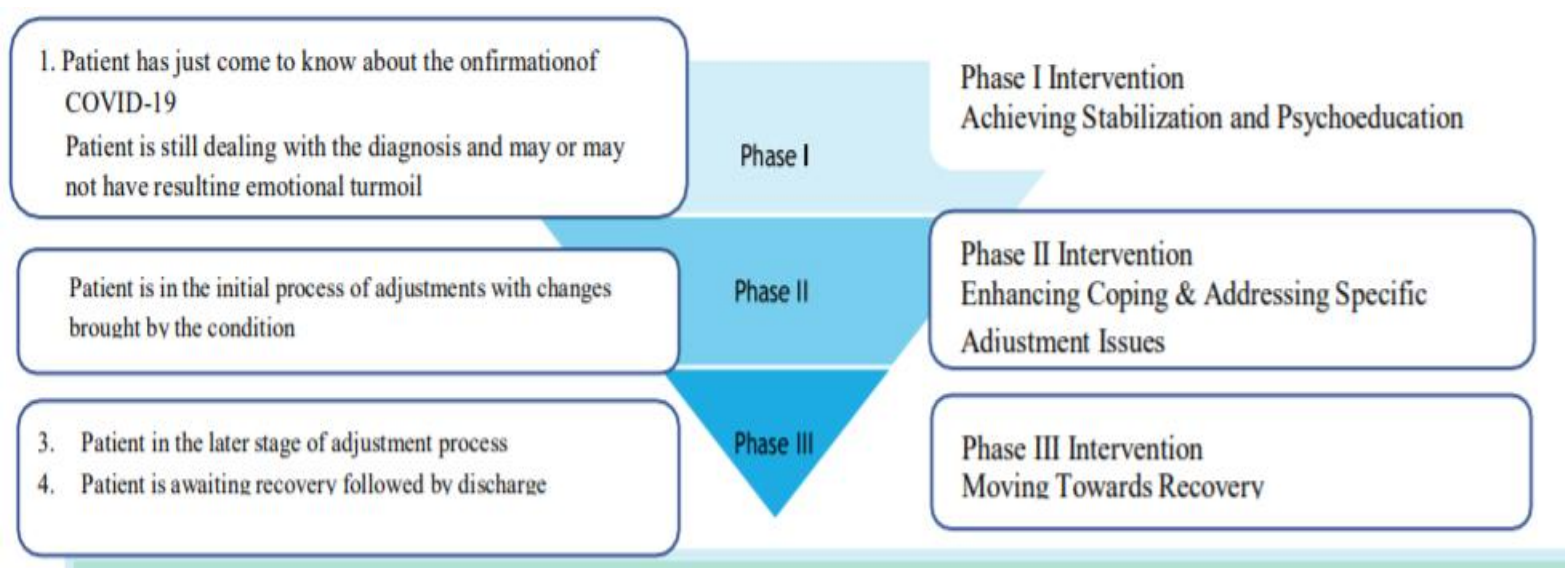

(Source: https: //www.icmr. gov. in/)

\section{Phases}

Phase I Intervention: Achieving Stabilisation \& Education

The primary goal of Phase I is to enable the patient to overcome some of the impending emotional anxiety and distressful thoughts that could be experienced due to diagnosis of COVID-19. The predominant techniques are as follows:

a) Validation of Feelings: This involves recognising and understanding patient's thoughts, feelings etc.

b) Acceptance of Feelings: The process of acceptance includes listening out and validating the concerns/distress of the patient.

c) Management of Initial Emotional Disturbance: A patient having COVID-19 may feel emotionally overwhelmed during various stages of illness. Even if the emotional disturbance is frequent and severe, it can be handled by avoiding the urge to let it go, emphasizing on the phasic nature of emotion, allowing the patient to express emotions, practicing relaxation technique and accumulate positive emotions.

d) Health education: Counselor shall explain about the aspects of disease, current epidemic situation, recovery period, quarantine stay facilities, treatment process etc keeping in mind the patient's level of acceptance.

Phase II Intervention: Enhancing Coping \& Addressing Specific Adjustment Issues

By the end of Phase I, a COVID 19 patient comes out well regarding their diagnosis situation and become aware of the challenges associated with it and various ways of addressing it. In Phase II the focus is on actual implementation of planned actions into day - to - day functions. The main purpose of the intervention at this stage is to help patients 
deal with COVID-19 related adjustment issues as wells as enhance their coping skills. The techniques are as follows:

a) Reducing Psychological Impact due to Isolation.

b) Dealing with Imaginary Anxieties.

c) Mitigating Fear of Death.

d) Managing Concerns about the Well - Being of Family Members.

e) Enhancement of different types of coping mechanisms.

Phase III Intervention: Moving Towards Recovery

When the patient is in the final stage of recovery, different sets of techniques need to be employed.

- Supportive Counseling: During the uncertain period of recovery, patient may show a sign of losing hope therefore during those times, it is important to provide supportive counseling to the patient so that he or she continues to make progress.

- Practicing Self - Compassion: Here the Counselor should advise the patient to show more self compassion and let others try their best to help the patient.

- Building Hope: This includes discussion on positive aspects of life. Counselor should impress upon the patient that future is not that bleak as it appears and there is prospect of good times ahead after the recovery.

- Termination: The changes discussed in the various sessions shall be summarized and drafted into an action plan.

\section{Role of a nurse}

- Establishing a Therapeutic Relationship

- Provide emotional support

- Listen carefully and attentively

- Respect the patient

- Encourage to reveal the emotions

- Help them to deal their worries in a healthy way

\section{Conclusion}

The techniques mentioned in the guidelines constitute the Intervention's core elements and they do not anyway restrict a Counselor from adopting better approaches for a patient. A counselor/volunteer may often adopt overlaps between techniques while some technique may be used more often than others or in different sequences. Thus, health care professionals are encouraged to modify, adapt and tailor the pre - set intervention approaches based on the guidelines to fit the specific needs of the patients. Nurses on frontline workers can impart psychosocial counseling to patients and family members in the clinical and community settings

\section{References}

[1] Guidance Document for Psychosocial Counseling for COVID-19 Positive Patients and their Family Members, available at https: //www.icmr. gov. in/, on 2021, downloaded on 27/07/2021.

[2] Guidelines to be followed on detection of suspect/confirmed COVID-19 case in a non COVID Health Facility available at https: //www.mohfw. gov. in/ on 2020, downloaded on 27/07/2021.
[3] Mental health and psychosocial support during COVID19 available at https: //apps. who. int/ on 2021, downloaded on 28/07/2021.

[4] Psychosocial Support during the COVID-19 pandemic available at https: //tiss. edu/ on 2021, downloaded on30/07/2021.

[5] Psychosocial response to COVID-19 pandemic in India: Helpline Counselors' experiences and perspectives available at https: //rcni. com/ on 2021, downloaded on 30/07/2021.

[6] Psychosocial counseling for Covid 19 https: //www.nimhans on 2021, downloaded on 18/08/2021. 